\title{
Détentions et déportation à la frontière entre le Mexique et les Etats-Unis (partie 2)
}

\section{Samuel Schmidt}

\section{(2) OpenEdition \\ Journals}

\section{Édition électronique}

URL : http://journals.openedition.org/conflits/623

DOI : $10.4000 /$ conflits.623

ISSN : $1777-5345$

Éditeur :

CCLS - Centre d'études sur les conflits lilberté et sécurité, L'Harmattan

\section{Édition imprimée}

Date de publication : 15 octobre 1996

ISSN : 1157-996X

\section{Référence électronique}

Samuel Schmidt, « Détentions et déportation à la frontière entre le Mexique et les Etats-Unis (partie 2) », Cultures \& Conflits [En ligne], 23 | automne 1996, mis en ligne le 15 mars 2006, consulté le 30 mars 2021. URL : http://journals.openedition.org/conflits/623 ; DOI : https://doi.org/10.4000/conflits.623

Ce document a été généré automatiquement le 30 mars 2021.

Creative Commons License 


\title{
Détentions et déportation à la frontière entre le Mexique et les Etats-Unis (partie 2)
}

\author{
Samuel Schmidt
}

1 La loi migratoire des Etats-Unis mise en perspective Indépendamment de leur rôle dans les deux systèmes économiques les migrants dépourvus de papiers d'identité sont perçus comme criminels et souffrent des abus de la police des deux pays ${ }^{1}$. Les migrants avec et sans papiers souffrent des vexations des agents migratoires américain et de la «border patrol». Ils souffrent également des bandes de délinquants (gangs), des criminels et des groupes racistes. A San Diego, un groupe de jeunes allait à la frontière pour « chasser » des Mexicains. Dans le cas des Etats-Unis la philosophie qui soutient cette position politique s'appuie d'une part sur une longue série d'essais visant à restreindre l'immigration afin de ne pas rompre la suprématie blanche, plus connue sous les initiales WASP (White Anglo Saxo Protestant), et d'autre part pour réglementer la force de travail. Rappelons qu'au début du XXe siècle on tenta également de limiter l'impact politique de la migration européenne et on ouvrit les portes à l'immigration mexicaine ${ }^{2}$. De toute façon la loi sert à traduire juridiquement un sentiment national non dépourvu d'influence idéologique ou d'une distorsion d'un véritable objectif. Selon Kitty Calavita ${ }^{3}$ la loi occulte les contradictions. Dans le cas de la loi migratoire c'est le facteur d'exploitation du travail qui est occulté. Il en résulte que la " criminalisation » du travail et par conséquent de l'immigration font partie du même phénomène philosophico-politique. De plus on utilise fréquemment la condition de migrant comme une circonstance aggravante dès qu'il y a un soupçon de criminalité. Selon Daniel Wolf un travailleur sans papier d'identité sera plus facilement détenu sans caution qu'un citoyen en règle ${ }^{4}$. De plus, dans la zone de San Diego, la police prétend que les «sans papier " sont coupables de crime, même s'ils sont souvent eux-mêmes la cible des crimes. Ajoutons à cela la définition politique d'une zone « vide » considérée comme no man's land ainsi que les activités clandestines qui se succèdent faute de surveillance et protection. Tout ceci engendre ce que Javier Valenzuela appelle un écosystème de violence frontalière. 
2 Les antécédents légaux Les antécédents sur le problème des travailleurs étrangers aux Etats-Unis, se mélangent d'une certaine manière avec les tentatives pour sanctionner les employeurs. Même si on a discuté au XXe siècle plus ouvertement de la question des sanctions, il est évident que ces dernières étaient utilisés par les législateurs comme soutien de la loi. La première initiative remonte à 1885, quand la loi fut adoptée et connue sous l'«Alien Contract Labor Law». En 1917, la politique de restriction migratoire fut consolidée par l'instauration d'un examen d'écriture et de lecture, ainsi que par la création d'une zone d'exclusion pour les Asiatiques, sauf le Japon. Des pouvoirs discrétionnaires étaient donnés aux autorités migratoires pour admettre les membres des groupes exclus. En 1918 on adopta la loi qui permettait d'exclure et déporter les étrangers subversifs, on l'appela Anarchist Act. On imposa en 1921 le système des quotas afin de ralentir le flux migratoire postérieur à la première guerre mondiale. Ce système qui fut adopté comme loi en 1924, freina les immigrants du Sud et d'Europe de l'Est. Ces règles restèrent en vigueur jusqu'en 1952. En 1938, on y apporta un amendement, qui devint le Fair Labor Standards Act. Il incluait des sanctions à l'encontre de ceux qui employaient des étrangers sans autorisation de travail. En 1952, l'introduction, le transport et l'accueil volontaire de travailleurs sans papier devint un acte illégal ${ }^{5}$ puni soit par une amende d'un maximum de 2000 \$ soit par un emprisonnement qui pouvait aller jusqu'à cinq ans, ou même par les deux sanctions. Cependant cette mesure ne concernait pas l'emploi des travailleurs sans papiers. Au début des années soixante-dix on essaya d'inclure cette mesure et bien que les sanctions à l'encontre des employeurs rencontrèrent un écho favorable lors des législatures, le Sénat les rejeta. Les Président Ford et Carter établirent alors une législation fixant des peines pour les employeurs «conscients » d'avoir embauché des travailleurs sans papiers. Le Président Carter fit une proposition de loi relative à l'augmentation des contrôles frontaliers, mais on ne lui donna aucune suite lors de la séance du Congrès. Durant les années quatre-vingt le Sénateur Simpson et le représentant Mazzoli organisèrent des audiences conjointes qui débouchèrent finalement sur la loi migratoire de 1983, qui ne fut cependant pas adoptée en raison des différends existant entre les législateurs. La discussion de la loi fut reprise en 1985 et adoptée l'année suivante comme Immigration Reform and Control Act (IRCA). Elle prévoit des sanctions pour les employeurs avec provision anti-discriminatoire. La principale contribution apportée par cette loi est sans aucun doute la «criminalisation » du travail et la connotation criminelle introduite dans la définition de la migration. La meilleure preuve du renforcement de cette définition est la loi criminelle de 1994 qui prévoit l'allocation de fonds pour l'augmentation du nombre d'agents luttant contre l'immigration ${ }^{6}$. Depuis la tentative de 1952 l'innovation juridique réside dans l'établissement de peines criminelles pour ceux qui protégeraient ou emploieraient consciemment des travailleurs sans papiers. L'IRCA établit une peine de $3000 \$$ et/ou six mois de prison. Pour les récidivistes on établit des sanctions criminelles. Cependant le service d'immigration durant la première année après l'adoption de la loi se consacra à instruire les employeurs à propos de cette loi et à leur dire comment éviter les sanctions, ce qui consistait en pratique à les entraîner à détecter les faux papiers et à dénoncer les sans papier. L'INS évite de sanctionner les employeurs préférant donner une leçon aux immigrants récidivistes et se montre même prêt à négocier les amendes ${ }^{7}$. Le fait que ni l'INS ni la « border patrol » n'aient un nombre suffisant d'agents pour appliquer les sanctions démontre peut-être le peu d'enthousiasme à « déranger » les hommes d'affaires, préférant ainsi sanctionner les 
immigrants par des actions, violant souvent les droits de l'homme. Déportations Les antécédents légaux de déportations se trouvent dans l'Alien Act de 1798 selon lequel le Président peut exiger l'expulsion de n'importe quelle personne qu'il considère comme dangereuse. En 1888 le Congrès sanctionna la déportation de travailleurs étrangers employés durant l'année de leur entrée au pays. Ce pouvoir d'expulsion s'étendit à tous les étrangers entrés en transgressant la loi. Le statut migratoire a maintenu des directives similaires bien qu'étendant la durée qui établissait certaines restrictions pour son application. Finalement ces restrictions furent supprimées lorsque la loi fut adoptée en 1952. Les causes qui déterminent la déportation étaient définies par une mauvaise conduite après l'entrée dans le pays. En 1910 la prostitution était mentionnée bien que le Congrès ait continué à élargir la définition de mauvaise conduite. Cependant il est clair que la faute la plus importante est l'entrée dans le pays sans inspection. D'autres raisons se réfèrent aux activités criminelles subversives, immorales, aux activités de contrebande et de trafics de drogue. En plus de la déportation il existe dans la loi l'interdiction du territoire dont les raisons sont similaires à celles de la déportation et qui autorisent tout simplement le service de l'immigration à interdire l'entrée du pays à celui qui a commis les crimes mentionnés. Il semble y avoir consensus sur le fait que la déportation est une sanction dont la sévérité dépasse la peine criminelle la plus sévère et la Cour Suprême caractérisa la déportation comme une "mesure grave qui équivaudrait au bannissement ou à l'exil » 8. L'organisme chargé de formuler et de superviser la politique migratoire est le Département de la Justice, dont dépend le Service de l'Immigration et de la Naturalisation (INS). A sa tête se trouve une commission dont la composition est proposée par le Président et approuvé par le Congrès. La fonction policière au sein de l'INS est à la charge de la «border patrol ». La « border patrol » comme l'INS arrêtent les immigrants sans papiers. Ils ont à leur disposition différents centres de traitements et de détention des prisonniers. Il existe 34 points de passages à la frontière, chacun ayant son propre centre de traitement des prisonniers. Sur les 17 centre de détention du pays, 7 se trouvent à la frontière du Mexique. Un seul se trouve dans un camps militaire, sur une base de la garde côtière de Boston. La répartition des centres de détention par Etat est la suivante :

Centres de rétention par Etat Arizona 1 Californie 2 Texas 4 Hors de la Frontière Colorado 1 Floride 1 Louisiane 1 Massachussetts 1 New York 2 Porto Rico 1 Washington 1 Washington D.C. 1

4 L'INS possède et dirige neuf de ces centres. Elle en possède quatre autres qui sont gérés par le bureau des prisons, deux appartiennent et sont dirigés par ce dernier et un autre est situé dans un hôpital de Washington D.C., dirigé en collaboration avec un service de santé publique. Le processus de déportation peut s'effectuer de deux manières différentes: soit par rapatriement volontaire, soit après audience. L'option du rapatriement volontaire n'est possible que pour les Mexicains situés dans la frange frontalière, car si le détenu la choisit on le ramène à la frontière et ce dernier repart à pied de sa propre volonté. Dans ce cas on n'intente aucune action judiciaire contre le détenu bien que celui-ci ait transgressé la loi en traversant la frontière sans papiers. C'est sans doute pour cette raison que l'on enregistre un nombre élevé de traversées car nombre de ces rapatriés essayent de retraverser la frontière dès qu'ils se trouvent au Mexique. Pour la petite histoire on raconte que nombre de rapatriés sont détenus plus de cinq fois en un jour et même souvent plus. Le détenu peut aussi solliciter une audience de déportation.devant le juge de l'immigration on ne lui procure pas d'aide 
légale bien qu'il ait le droit d'en demander une. Les immigrés sont souvent confinés dans des camps de détention ce qui rend difficile, l'accès à l'assistance légale. De plus si l'on réalise que la plupart de ces immigrants sont extrêmement pauvres il semble complètement invraisemblable de penser qu'ils puissent recourir à l'assistance d'une aide légale. A tel point que nombre de victimes des abus de la «border patrol» ou de l'INS sont prises en charge par des organisations philanthropiques ou politiques. Dès qu'un délit est constaté, la position des juges face aux immigrants est beaucoup plus sévère. Non seulement les cautions sont plus élevées mais le pourcentage qu'il faut payer pour la caution est également beaucoup plus important. Asile politique Un détenu peut solliciter l'asile politique grâce à plusieurs procédures. a) Dès qu'il est détenu par une autorité migratoire il a droit à une audience d'asile. Si celle-ci est refusée, le processus de déportation commence. $99 \%$ des demandeurs d'asile politique ne sont pas détenus, à l'exception des Chinois. Le détenu a le droit de demander l'assistance d'un avocat bien que l'Etat ne lui procure pas d'avocat public. Avant avec l'asile politique la personne recevait un permis de travail, selon certaines sources, ceci entraîna certains abus et une certaine corruption, car les avocats présentaient la demande d'asile pour obtenir le permis de travail et ensuite le candidat à l'asile ou bien ne se présentait pas aux audiences, ou attendait patiemment car il n'y avait pas un nombre suffisant de juges pour s'occuper de toutes les requêtes. Actuellement il reste en instance environ un demi million de cas. Dans le cas du Mexique, les demandes d'asile ont augmenté d'une façon spectaculaire, bien que la position négative des autorités migratoires américaines ne change pas. La raison en est la définition politique des pays établie par le département d'Etat: dans les pays considérés comme démocratiques, tels que le Mexique, les citoyens ne sont pas candidats à l'asile. Pour le gouvernement des Etats-Unis il y a au Mexique des élections systématiques et même des partis d'opposition au pouvoir ce qui est suffisant pour démontrer le caractère démocratique du régime. De toutes les façons, on notait 122 demandes d'asile en 1990, pour parvenir à un maximum de 9791 en 1994. On atteint 5292 demandes en 1995, pour un total sur six ans de 22404. 37 ont été acceptées, 5936 refusées, 5817 dossiers ont été classés, il reste 9124 cas. tableau III : Demande d'asile politique de Mexicains ${ }^{9}$

Total Demandes mexicaines

6 Demandes \% admis Demandes \% du total Acceptées \% admis 19907363714.71220 .200 19915631033.61880 .300199210396437 .66140 .600199314416621 .863974 .400 1994148348 n.d. $97916.660 .061995^{10} 73500$ n.d. 52927.2310 .58

7 Le pourcentage de demandes acceptées est toujours aussi faible. En 1993 on accepta 21.8\% des cas, mais aucun mexicain, tandis qu'en 1995 le pourcentage ne dépassait pas $0.5 \%$. Contrairement aux autres pays comme Cuba pour lequel l'asile est accordé presque automatiquement, pour le Mexique le processus est très lent est complexe. En effet, dans les cinquante dernières années un seul cas fut admis, celui d'un politicien de l'Etat frontalier de Chihuahua qui durant les années soixante dix emporta la victoire contre le PRI, ce qui lui valut une implacable persécution, accusé de trafic de drogue, torturé par la police il décida de quitter le pays ${ }^{11}$. Apres un jugement tardif, on lui concéda l'asile politique, mais jusqu'à maintenant l'INS a fait tout ce qui est en son pouvoir pour le rapatrier, au point de lui refuser un visa permanent. L'INS déclare d'une façon non officielle qu'il est trafiquant de drogue, bien qu'aucune preuve n'ait été produite. L'ironie de l'Histoire, est que le commandant qui le tortura et servit d'informateur pour le livre qui l'accusa de trafiquant, a fui le Mexique $^{12}$. Le 
gouvernement sollicita son extradition des Etats-Unis laquelle fut refusée, en partie parce que les témoins, peut-être par crainte, décidèrent de ne pas témoigner. Si l'image des Etats-Unis au Mexique comme refuge contre la persécution politique est en train de changer, aux Etats-Unis elle change également mais dans le sens contraire, puisque l'on se dirige vers une politique de contrôle de la frontière, avec une combinaison d'opérations policières, de constructions, de clôtures et l'utilisation d'une technologie de pointe pour détecter rapidement les immigrés sans papiers. En ce qui concerne la politique, la position de soutien du gouvernement mexicain, consiste a nier " a priori » les requêtes d'asile se basant sur l'ouverture politique mexicaine et en minimisant la persécution et la répression mexicaines. On établit simultanément des barrières légales à l'arrivée des réfugiés politiques.

tableau IV : Murs frontières à la frontière américano-mexicaine

Etats Villes Californie/Baja California San Diego/Tijuana Calexico/Mexicali Campo/ Tecate Arizona/Sonora Nogales/Nogales Naco/Naco Douglas/Agua Prieta

Le fait que le nombre de demandes d'asile ait augmenté de $942 \%$ d'une année sur l'autre est sans importance pour le gouvernement des Etats-Unis qui soutient le gouvernement mexicain afin que celui-ci freine ses propres citoyens. C'est ainsi que l'on ferme le cercle qui propose l'intégration économique avec le Mexique mais sans les mexicains. En 1993 l'administration Clinton approuva l'opération "Blocus ", dénommée par la suite « Hold the line» entre El Paso et Ciudad Juarez. Elle fut justifiée par le fait qu'il était plus efficace de freiner les migrants avant qu'ils ne passent la frontière, plutôt que de les poursuivre. Sans compter que la politique de persécution à l'intérieur du territoire américain, avait provoqué des nombreuses plaintes pour atteintes aux droits de l'homme ${ }^{13}$. Ces plaintes étaient adressées aux autorités locales et avaient fait fleurir de nombreuses associations de défense des droits de l'homme, ouvertement opposées aux patrouilles frontalières, ce qui avait détérioré les relations avec le gouvernement mexicain. De plus, on justifia cette mesure en prétextant qu'il fallait freiner les criminels qui passaient du Mexique aux Etats-Unis, ainsi que les Mexicains qui abusent du système d'assistance. «Freiner les criminels » a constitué l'argument essentiel pour favoriser la construction de clôture à la frontière, à tel point que nombre de personnes parlent déjà du nouveau mur de Berlin à la frontière entre les Etats-Unis et le Mexique. L'opération «blocus» fut efficace et réduisit substantiellement le nombre de prisonniers, de sorte que l'on décida avec l'appui total de Washington de l'étendre à l'Arizona et à la Californie. A San Diego, en Californie, on l'appelle «l'opération «Gate Keeper » et en Arizona l'opération « Safeguard. Pour appuyer la politique de fermeture de frontière, le gouvernement de Clinton promulgua une loi contre le crime, dans laquelle on donnait une grande importance à l'immigration clandestine, en décidant notamment d'augmenter le nombre d'agents de la «border patrol» et de l'INS, la construction de clôture et de critères différents pour mesurer l'efficacité de la «border patrol ». Autrefois la «border patrol » contrôlait un grand nombre de prisonniers, aujourd'hui ils s'avèrent moins nombreux. On a observé une réduction importante de prisonniers dans les zones contrôlées par des "opérations blocus" et une augmentation dans les zones où il n'y en avait pas. Une ou plusieurs explications combinées peuvent être avancées: Le nombre d'immigrants sans papiers n'était pas aussi élevé que le chiffre annoncé officiellement; les immigrants se déplacent vers les zones moins protégées et les arrestations augmentent. Les méthodes de la «border 
patrol » sont efficaces. Il y a un augmentation de l'immigration mexicaine en réponse à l'actuelle crise économique.

11 On peut remarquer une intense campagne de relations publiques de la part des fonctionnaires migratoires, comme par exemple de nombreuses visites de la commissions de l'INS à la frontière et la formation de divers comités de surveillance de l'INS et de la «border patrol». Le Consul général du Mexique de El Paso participe au "Civilian Advisory Panel to The US Attorney general to review complaint and procedure misconduct of INS employees ». La concordance des points vues démocrates et républicains au sujet de l'immigration est très particulière. La proposition 187 qui restreint l'aide aux immigrants sans papiers a été adoptée en Californie sous l'autorité d'un gouverneur républicain, alors que l'administration démocrate avait déjà durci sa position, quoique plus discrètement. Cependant les deux partis ont trouvé commode d'accuser les Mexicains d'être la cause de la crise économique et fiscale des Etats-Unis. Ils ont pris ces derniers comme boucs émissaires, d'autant plus faciles à utiliser qu'ils méconnaissent les lois américaines, que les réseaux politiques importants d'aide aux migrants sont peu nombreux, mais également à cause de la faiblesse du gouvernement mexicain, qui au lieu d'entamer un processus d'intégration économique, a laissé de côté l'étude bilatérale des questions sensibles, parmi lesquelles celles immigrants sans papiers est sans doute la plus importante.

12 Conclusion Les décisions gouvernementales entre le Mexique et les Etats-Unis destinées à ralentir l'immigration ont échoué. L'installation d'usines de sous-traitance à la frontière est un échec. Ces dernières ont engendré la création de 500.000 emplois mal payés, intègrent à peine $2 \%$ de produits nationaux (essentiellement des empaquetages). En outre elles ont causé de graves dommages au milieu ambiant en ne respectant pas les règlements de protection de l'environnement ${ }^{14}$. Ces entreprises ont créé de gigantesques cordons de misère tout le long de la frontière Mexique-Etats-Unis. $60 \%$ de la ville de Juarez, ville frontalière avec El Paso, Texas, abritent des implantations irrégulières. Elles n'offrent donc certainement pas les services minimum tels l'eau courante, le tout-à-l'égout, etc. Ces cordons ne freinent ni la migration, ni les microbes ou les bactéries et la pollution qui n'exigent ni passeport ni visa, et c'est ce qu'aucune autorité migratoire ne réussira à arrêter, même par erreur. La migration, même volontaire, répond à un situation économique complexe. Les cyniques prétendent qu'il s'agit d'un processus de reconquête silencieuse des territoires que le Mexique perdit au XIXe siècle, d'autres prétendent que c'est le véritable référendum de la politique économique du régime. Bien sur, la migration mexicaine a augmenté grâce à l'existence des réseaux de soutien et d'échange qui facilitent l'absorption, l'emploi et une meilleure compréhension de la société dans laquelle les migrants s'insèrent, mais en réalité ils abandonnent leur pays car les conditions de vie sont intolérables. On indique que certains Mexicains émigrent en acceptant un réduction de leur statut social. On a vu des femmes programmeurs d'ordinateurs accepter des places d'employées de maison, ou des maîtresses d'école qui émigrent pour faire la cueillette. Le Mexique est en train de gâcher l'énorme investissement destiné à augmenter le niveau de vie de sa population. Dans l'immigration actuelle on trouve des professionnels, des enseignants et des scientifiques qui abandonnent le pays, recherchant des revenus plus élevés et dont la qualification servira aux Etats-Unis. Si cette tendance continue le Mexique deviendra à l'avenir une société divisée entre une élite bien préparée et une masse non préparée puisque les couches sociales moyennes trouvent un salaire plus attirant au Nord. La solution est complexe et nécessite plus que de simples mesures policières. Elle 
exige l'assistance de tout ceux impliqués dans la formation de ce phénomène, c'est à dire les entreprises mexicaines, étrangères et multinationales qui ont bénéficié de l'exploitation de ces individus qui maintenant ont besoin d'émigrer. Il serait plus juste qu'ils coopèrent pour alléger cette charge qu'ils ont créée, et ce par un accord similaire à celui conclu lors la réunion de Rio, où il a été décidé que ceux qui détérioraient l'environnement devaient réparer les dommages qu'ils avaient créés. Transférons ce concept aux êtres humains, nous devons exiger que ceux qui ont causé la misère humaine paient pour réparer les dommages. L'étude de Vernez et Ronfeldt ${ }^{15}$ suggère qu'une augmentation de l'immigration affecte d'une façon négative le niveau du salaire. Ce qui veut dire que l'immigration mexicaine joue un rôle fondamental dans la réduction des salaires, ce qui permet de maintenir une inflation minime et de soutenir la compétitivité des Etats-Unis. Ainsi les abus infligés aux Mexicains par les Etats-Unis ont permis de réduire encore plus l'inflation. Cependant les Etats-Unis présentent les migrants mexicains comme des délinquants. Ainsi, on constate une discrimination contre les Mexicains dans chaque aspect de la société jusqu'à la militarisation de la frontière entre les deux pays avec les excès que cela entraîne de la part de la "border patrol ", et la création d'une zone de crime qui étend ses effets jusqu'en Amérique Centrale, ou les "polleros » ${ }^{16}$ mettent des petites annonces dans le journal, trompant ainsi et brimant les gens pauvres. Les Etats-Unis sont la cause de l'immigration sans papiers et doivent prendre part à l'élaboration d'une solution. Le Southwest US-Mexico Border Policy Group créé à El Paso au Texas suggéra pour réduire le niveau de la violence frontalière un déplacement des contrôles migratoires à 25 miles vers le Nord, la frange frontalière deviendrait ainsi une zone d'amortissement où la force du travail s'écoulerait librement. Inutile de préciser que l'INS et la « border patrol » s'opposèrent avec force à ce projet. Pour paraphraser l'Ambassadeur du Brésil au Mexique, Carlos Augusto Santos Neves : il faut envisager la migration comme la solution car le problème est la misère extrême et la disponibilité d'emplois mal payés ${ }^{17}$. Le Mexique et les EtatsUnis doivent trouver une solution permanente qui appréhende l'immigration comme un facteur naturel. Le Mexique tente en même temps d'élever son niveau économique en donnant la priorité à l'amélioration de la qualité de la vie. En attendant la solution de l'immigration doit être bilatérale. La communauté internationale a développé un statut de réfugié politique pour les personnes persécutées (en raison de leurs idées politiques notamment), maintenant elle doit développer un statut de réfugié économique pour ceux qui furent exploités par les systèmes économiques dans lesquels ils s'insèrent. L'existence d'un pays et un sous-continent divisé entre une poignée de multimillionnaires et une masse de «crève la faim » n'est pas seulement immorale, c'est aussi invivable. Les Etats-Unis ont la possibilité de mieux utiliser la progression économique mexicaine, pour intégrer de la sorte une zone sans pauvreté qui améliorerait le niveau de vie au Mexique et qui diffuserait jusqu'en Amérique Centrale. Les autorités migratoires mexicaines révèlent, que l'on trouve 42.000 personnes provenant du Salvador munis de papiers d'identité dans le pays, ce qui signifie probablement 500.000 sans papiers. Le Mexique constate à sa frontière Sud les mêmes problèmes que les Etats-Unis observent à leur frontière Sud. Les solutions partielles et à retouches ne marcheront pour aucun pays tant qu'ils ne trouveront pas de solutions globales qui tirent l'Amérique Latine de son retard endémique. La nouvelle vague antimigratoire, et plus spécifiquement anti-mexicaine, qui traverse les Etats-Unis, avec la Californie pour centre, est davantage due à une combinaison de l'escalade républicaine qui a incendié le pays contre les démocrates en exploitant les peurs par une 
redéfinition des ennemis. Campagne qui sert a préparer la campagne présidentielle de 1996, avec le mécontentement lié à une récession prolongée initiée par les républicains et qui, bien que résorbée par les démocrates, n'a pas encore eu de retombées psychologiques sur l'américain moyen. Les Etats-Unis se trouvent au centre, d'une importante confrontation idéologique qui redéfinit le rôle de l'Etat et probablement les relations entre la société et la société politique. Malheureusement, les deux positions extrêmes de la polémique ont concentré leurs énergies sur les immigrants, avec ou sans papiers d'identité, ou ces derniers sont sans aucun doute les plus vulnérables. Cet état de fait n'aide évidemment pas à planifier un futur meilleur et encore moins à planifier des relations cordiales entre les Etats-Unis et leurs voisins du Sud.

Revenir à la partie précédente

\section{NOTES}

1. Le gouvernement mexicain (1988-1994) créa le groupe Beta dirigé par le Secretariat du Gouvernement, formé par des policiers choisis dans plusieurs corps policiers pour s'attaquer à la criminalité dans la terra de nadie entre Tijuana et San Diego. La première mesure consista à interdire l'entrée des policiers dans la zone, la criminalité fut réduite considérablement.

2. Calavita Kitty, op. cit., p. 10.

3. Calavita Kitty, op. cit., p. 6-9.

4. Wolf, Daniel, Undocumented Aliens and Crime. The Case of San Diego County, San Diego, University of California, San Diego Center for U.S.-Mexican Studies Monograph Series, 29., 1988, p. 1.

5. Beradi Gayle K., "The Ramifications of IRCA Employer Sanctions : A Case Study of an East Texas Community ", Rio Bravo, Vol. II, nº 2 Spring, 1993, pp. 61-84.

6. Administration Plans Hard Reform, El Paso Times, Enero 10, 1995, p. 3 A.

7. La description de la position de l'INS est fondée sur un entretien avec Alfred Giugni Directeur du district à El Paso,(9 nov. 1994).

8. Gordon Gittel, Gordon Charles, Immigration Law and Procedure, New York, Mathew Bender and Co., 1991, p. 71-72.

9. Source : U.S. Department of Justice, Immigration an Naturalization Service, INS Fact Book, U.S Government Printing Office, 1993, p. 16 ; U.S. Department of Justice, 1993 Statistical Yearbook ofthe Immigration and Naturalization Service, september 1994, p. 85.

10. De octobre 1994 à mars 1995.

11. Shmidt, Samuel, « De policias Fronterizos », UnomasUno, Dic. 22, 1994, p.9.

12. Poppa, Terrence E, Druglord: The Life and Death of a Mexican Kingpin : a true story, New York, Pharos Book, 1990.

13. America's Watch, Brutality Uncheked : Human Rights Abuses along the U.S. border with Mexico, New York, Human Rights Watch, 1992

14. George Baker, «Mexican Labor is not Cheap », Rio Bravo, n 1, Fall, 1991.

15. Vernez Georges, Ronfeldt David, op. cit. 
16. On appelle ainsi ceux qui introduisent les gens sans papier.

17. L'Ambassadeur se referrait aux favelas comme solution, le problème est le manque de logement. Il ne niait pas que les favelas ne réunissaient pas les conditions minimales d'un logement digne.

INDEX

Index géographique : Etats-Unis, Mexique

Mots-clés : centre de rétention, expulsions, frontières 